\title{
Parental origin and mechanisms of formation of cytogenetically recognisable de novo direct and inverted duplications
}

Institute for Medical Genetics, University of Zürich, Zürich, Switzerland

D Kotzot

M-J Martinez

A Baumer

F Binkert

L Brecevic

F Dutly

M Riegel

A Schinzel

Department of Medical Biology and Genetics, Akdeniz

University, Antalya, Turkey

G Bagci

S B Karaüzüm

G Luleci

Department of Pediatrics, University of Istanbul, Istanbul, Turkey

$S$ Basaran

Genetic Counselling Service, Bozen, Italy C Castellan

The Children's

Memorial Health

Institute, Warsaw, Poland

K Chrzanowska

A Gutkowska

M Krajewska-Walasek

Department of Medical Genetics, Children's Hospital Basel, Basel,

Switzerland and Aristogen GmbH, Ingelheim, Germany P Miny

Department of Medical Genetics, Children's Hospital, Ludwig Maximilian University München, Germany

S Schuffenhauer

H Seidl

Correspondence to: Dr Kotzot, Institut fü Medizinische Genetik, Universität Zürich, Rämistrasse 74, CH-8001 Zürich, Switzerland. Kotzot@medgen.unizh.ch

Revised version received 30 November 1999 Accepted for publication 8 December 1999

Dieter Kotzot, Maria-Jose Martinez, Gulseren Bagci, Seher Basaran, Alessandra Baumer, Franz Binkert, Lucrecja Brecevic, Claudio Castellan, Krystyna Chrzanowska, Fabrizio Dutly, Anna Gutkowska, Sibel Berker Karaüzüm, Malgorzata Krajewska-Walasek, Guven Luleci, Peter Miny, Mariluce Riegel, Simone Schuffenhauer, Heide Seidel, Albert Schinzel

\begin{abstract}
Cytogenetic, FISH, and molecular results of 20 cases with de novo tandem duplications of 18 different autosomal chromosome segments are reported. There were 12 cases with direct duplications, three cases with inverted duplications, and five in whom determination of direction was not possible. In seven cases a rearrangement between non-sister chromatids (NSCR) was found, whereas in the remaining 13 cases sister chromatids (SCR) were involved. Paternal and maternal origin $(7: 7)$ was found almost equally in cases with SCR (3:4) and N-SCR (4:3). In the cases with proven inversion, there was maternal and paternal origin in one case each. Twenty three out of 43 cytogenetically determined breakpoints correlated with common or rare fragile sites. In five cases, including all those with proven inverse orientation, all breakpoints corresponded to common or rare fragile sites. In at least two cases, one with an interstitial duplication (dup(19)(q11q13)) and one with a terminal duplication $(\operatorname{dup}(8)$ (p10p23)), concomitant deletions (del(8) (p23p23.3) and $\operatorname{del}(19)(q 13 q 13))$ were found.

(F Med Genet 2000;37:281-286)
\end{abstract}

Keywords: direct duplication; inverted duplication; parental origin; tandem duplication

Tandem duplications are direct or inverted duplications of genetic material, ordered one after the other. Cytogenetically recognisable tandem duplications are rare and comprise several megabases. The clinical phenotype of most cases is characterised by multiple congenital anomalies and developmental delay. Case reports of tandem duplications of almost all regions of human chromosomes have been reported ${ }^{1}$ and most of them have occurred de novo. Routine investigations include conventional karyotyping and fluorescence in situ hybridisation (FISH) with whole chromosome libraries. So far, mechanisms of formation and parental origin have not been investigated in a larger series. Mismatched pairing of homologues and unequal crossover between nonsister chromatids or sister chromatids in direct duplications, as well as three break rearrange- ments including a $U$ type rearrangement in inverted duplications, have been assumed. ${ }^{2}$ To the best of our knowledge, apart from a group of 16 cases with mono- and dicentric $8 p$ duplications exclusively formed in maternal meiosis, ${ }^{3}$ few cases with de novo tandem duplications have been investigated by molecular methods, for example, dup(5)(q11.2q14)pat, inv dup (6) (q22q23)pat, inv dup (6) (q24.3q27) pat, inv $\operatorname{dup}(7)(\mathrm{q} 21.2 \mathrm{q} 36), \quad \operatorname{dup}(19)$ (q13.2q13.4) mat, and $\operatorname{dup}(21)(\mathrm{q} 11.2 \mathrm{q} 22.3)$ mat. ${ }^{4-9}$ The dup(21)(q11.2q22.3)mat was mosaic with a normal cell line despite meiotic formation. ${ }^{9}$ In the group of $\operatorname{dup}(8 \mathrm{p})$ and in the case with inv $\operatorname{dup}(7)(\mathrm{q} 21.2 \mathrm{q} 36)$ concomitant deletions were found. ${ }^{3}$

Here, we report FISH and molecular findings in 20 cases of cytogenetically recognisable de novo direct or inverted tandem duplications of 18 different autosomal chromosome segments.

\section{Subjects and methods}

All patients were clinically evaluated by experienced clinical geneticists and referred to the Institute for Medical Genetics, Zürich, either for cytogenetic/molecular investigations or for clinical evaluation over a period of more than 10 years. It was not possible to receive further material for specific investigations in all cases. One patient (case 19) has been published in 1993..$^{10}$ Part of this study was presented at the 11 th Annual Meeting of the German Society of Human Genetics. ${ }^{11}$ Clinical data of the patients as well as parental ages of all cases are available from the authors on request.

Lymphocyte chromosome examinations were performed according to standard procedures (400-600 bands per haplotype). In most cases $\mathrm{Q}$ and $\mathrm{G}$ banded karyotypes were evaluated. Metaphase FISH procedures were also performed according to the manufacturer's instructions with standard protocols for whole chromosome libraries (ONCOR ${ }^{\circ}$ Inc, Gaithersburg) and in part with specific probes located at subtelomeric or telomeric loci. ${ }^{12}$ The latter were obtained from ATTC $^{\circ}$ (Rockville, MD). Briefly, purified DNA from clones was labelled with either biotin-16-dUTP (Boehringer Mannheim ${ }^{\circ}$, Mannheim, Germany) or digoxigenin-11-dUTP (Boehringer Mannheim ${ }^{\circ}$, Mannheim, Germany) by nick translation. FITC avidin and rhodamine antidigoxigenin were used to detect these probes. 
Analysis was performed using a Zeiss Axioplan epifluorescence microscope. Images were recorded by Photometrics CCD KAF1400 camera (Photometrics, Tucson, Ariz) and controlled with Smart Capture imaging software (Vysis ${ }^{\mathcal{O}}$ Inc, Downers Grove, IL).

Genomic DNA was isolated from blood by salt extraction. For Southern blot analysis DNA was digested, electrophoresed in agarose, transferred to nylon membranes, and hybridised with ${ }^{32} \mathrm{P}$ labelled probes before autoradiography. PCR analysis was performed as described by Dutly et al. ${ }^{13}$ Briefly, highly polymorphic (heterozygosity $>0.7$ ) and commercially available microsatellites (Research Genetics $^{\mathcal{C}}$ ) were run on a $6 \%$ polyacrylamide gel and visualised by silver staining. Between 200 and $500 \mathrm{ng}$ of DNA were amplified in a volume of $25 \mu \mathrm{l}$. PCR amplification was performed on a Perkin-Elmer 9600 with 32 cycles of 30 seconds at $94^{\circ} \mathrm{C}, 45$ seconds at $55-60^{\circ} \mathrm{C}$ for annealing, and one minute $20 \mathrm{sec}-$ onds at $72^{\circ} \mathrm{C}$ for extension. The products were mixed in an equal volume of urea loading buffer and loaded onto the gel. Dosage results were interpreted by visually comparing the intensity of both alleles.

In each uninformative case a minimum of 10 , but mostly more than 20 microsatellites were used. Altogether, examinations with more than 300 different microsatellites were performed. Three alleles in one or more markers were considered as representing meiotic formation and non-sister chromatid rearrangement (N-SCR). A maximum of two alleles in the patient (one maternal and one paternal) for all informative markers was interpreted to be a result of sister chromatid rearrangement (SCR), if the marker was heterozygous in the parent of origin and the other parent had a different allele. Maternal and paternal uniparental disomy were excluded by markers located upstream and downstream of the duplicated region (data not shown). Non-paternity was

Table 1 Cytogenetic and molecular results

\begin{tabular}{|c|c|c|c|c|c|}
\hline Case & Cytogenetic results & Orientation & $\begin{array}{l}\text { Estimated } \\
\text { deletion } \\
\text { size }(c M)\end{array}$ & $\begin{array}{l}\text { Parental } \\
\text { origin }\end{array}$ & $\begin{array}{l}\text { Formation } \\
\text { by } \\
\text { molecular } \\
\text { results }\end{array}$ \\
\hline 1 & $46, \mathrm{XX}, \operatorname{dup}(2)(\mathrm{q} 35 \mathrm{q} 37)$ & ? & 20 & pat & SCR \\
\hline 2 & $46, \mathrm{XX}, \operatorname{dup}(2)(\mathrm{q} 35 \overline{\mathrm{q} 37})$ & ? & 20 & mat & SCR \\
\hline 3 & $46, \mathrm{XX}, \mathrm{dup}(4)(\mathrm{p} 13 \overline{\mathrm{p} 16})$ & dir & 50 & pat & N-SCR \\
\hline 4 & $46, \mathrm{XX}, \operatorname{dup}(4)(\mathrm{q} 25 \mathrm{q} 33)$ & dir & 75 & mat & N-SCR \\
\hline 5 & 46,XY,dup(5)(p14p15.3)/46,XY & dir & 20 & $?$ & ? \\
\hline 6 & $46, \mathrm{XX}, \mathrm{dup}(5)(\mathrm{q} 22 \mathrm{q} 35)$ & dir & 60 & mat & N-SCR \\
\hline 7 & 46,XY,dup (6) (q21q22) & dir & 8 & mat & SCR \\
\hline 8 & $46, X Y, \operatorname{dup}(7)(\overline{\mathrm{q} 22} \mathrm{q} 31)$ & dir & 12 & mat & SCR \\
\hline 9 & 46,XX,dup (8)(p11p23), del(8)(p23p23.3) & inv & 35 & mat & N-SCR \\
\hline 10 & $46, X Y, \operatorname{dup}(8)(\mathrm{q} 22 \mathrm{q} 24.3)$ & inv & 48 & pat & SCR \\
\hline 11 & $46, \mathrm{XX}, \mathrm{dup}(9)(\mathrm{p} 13 \mathrm{p} 24)$ & dir & 17 & pat & N-SCR \\
\hline 12 & $46, \mathrm{XX}, \operatorname{dup}(9)(\mathrm{p} 13 \overline{\mathrm{p} 24})$ & dir & 17 & pat & SCR \\
\hline 13 & $46, X X, \operatorname{dup}(10)(\mathrm{q} 2 \overline{2 \mathrm{q} 2} 6)$ & dir & 37 & ? & SCR \\
\hline 14 & $46, \mathrm{XX}, \operatorname{dup}(11)(\mathrm{q} 13.2 \mathrm{q} 13.4)$ & ? & 8 & pat & N-SCR \\
\hline 15 & $46, \mathrm{XX}, \operatorname{dup}(12)(\mathrm{q} 24.1 \mathrm{q} 24.3)$ & dir & 39 & ? & SCR \\
\hline 16 & $46, X Y, \operatorname{dup}(14)(\mathrm{q} 21 \mathrm{q} 21)$ & ? & 9 & ? & SCR \\
\hline 17 & 46,XY,dup (15) (q13q13) & ? & 9 & ? & SCR \\
\hline 18 & $46, \mathrm{XX}, \operatorname{dup}(17)(\mathrm{p} 11 \mathrm{p} 13)$ & dir & 20 & pat & N-SCR \\
\hline \multirow[t]{2}{*}{19} & $46, X X, \operatorname{dup}(19)(\mathrm{q} 11 \underline{q} 13), \operatorname{del}(19)$ & & & & \\
\hline & $(\mathrm{q} 13 \mathrm{q} 13), \operatorname{del}(16)(\mathrm{q} 21 \mathrm{q} 21)$ & dir & 15 & mat & SCR \\
\hline 20 & $46, \mathrm{XY}, \mathrm{dup}(22)(\mathrm{q} 11.1 \mathrm{q} 13.1)$ & inv & 35 & ? & SCR \\
\hline
\end{tabular}

Breakpoints corresponding to common or rare fragile sites are underlined. mat $=$ maternal, $\mathrm{N}-\mathrm{SCR}=$ non-sister chromatid rearrangement, pat $=$ paternal, $\mathrm{SCR}=$ sister chromatid rearrangement. excluded by markers located on various other chromosomes (data not shown).

\section{Results}

Clinical findings in all patients (13 females, seven males) included multiple congenital anomalies, non-specific patterns of dysmorphism, and developmental delay. Details are available on request from the corresponding author.

The cytogenetic and molecular results of our study are summarised in table 1. Additional chromosomal material was visible on each evaluated metaphase in 19 cases. In one patient (case 5) mosaicism was detected. In most cases the abnormal banding pattern indicated a duplication of genetic material originating from the same chromosome. This was confirmed by FISH with whole chromosome libraries. In all cases only the abnormal chromosome and its normal homologue were completely painted and no further chromosome was involved. Chromosome analysis of all parents showed normal results $(46, \mathrm{XX}$ or $46, \mathrm{XY}$ ) with no abnormal banding pattern shown by GTG banded metaphases and in part by FISH with whole chromosome libraries. Therefore, all duplications had occurred de novo and a balanced insertion/deletion in one parent could be excluded. Inversion in three cases had already been shown by conventional banding. In some smaller duplications it was not possible to discriminate unambiguously between a direct and inverted duplication. Therefore, inverted duplications might be more frequent. Interstitial telomeres were not detected in the 12 cases $(1-3,5,6$, $9-13,15$, and 18) with terminal duplications, suggesting subtelomeric breakpoints or even small concomitant telomeric deletions. However, it was possible to verify this assumption by microsatellite analysis in only one case (case 9). The concomitant deletion in case 19 was detected by loss of heterozygosity of the microsatellites applied. Terminal markers displayed only one allele. However, since the subtelomeric FISH probe was present and most of the distally located microsatellites were not informative, an interstitial deletion was assumed.

The results of molecular investigations are summarised in table 2. There were seven cases each of maternal and paternal origin. A minimum of five markers clearly indicating different intensity without any contradiction regarding parental origin was required to define parental origin in cases with SCR (fig 1). Owing to lack of the required number of informative markers, evaluation of parental origin was not possible in six cases.

Formation by N-SCR was inferred in seven cases (fig 2). Maternal origin was found four times and paternal origin three times. In the remaining 13 cases the distribution of the parental alleles indicated a SCR. A meiotic origin was found in seven cases (fig 2). In three cases with an inverted duplication, paternal SCR was found twice and maternal N-SCR once. In two cases, a concomitant deletion was detected, one was telomeric (del(8) 
$(\mathrm{p} 23 \mathrm{p} 23.3))$ in a case with an inverted duplication $(\operatorname{dup}(8)(\mathrm{p} 10 \mathrm{p} 23))$, the other (del(19) (q13q13)) was interstitial with a direct duplication $(\operatorname{dup}(19)(q 11 \mathrm{q} 13))$. In 10 cases one breakpoint and in five additional cases both breakpoints of the duplications were localised to the same cytogenetic band as common or rare fragile sites.

Table 2 Results of investigated markers mapping to the duplicated regions

\begin{tabular}{|c|c|c|c|c|c|}
\hline \multicolumn{2}{|c|}{$46, X X, d u p(2)(q 35 q 37) p a t$} & \multicolumn{2}{|c|}{$46, X X, d u p(2)(q 35 q 37) m a t$} & \multicolumn{2}{|c|}{$46, X X, d u p(4)(p 13 p 16) p a t$} \\
\hline Marker & $M / P / F$ & Marker & $M / P / F$ & Marker & $M / P / F$ \\
\hline $\mathrm{D} 2 \mathrm{~S} 371$ & $a b / b / b c$ & D2S1391 & $\mathrm{cd} / \mathrm{b} d / \mathrm{ab}$ & D4S43 & $a b / a b / b$ \\
\hline D2S355 & $\mathrm{ac} / \mathrm{a} d / \mathrm{bd}$ & D2S118 & $\mathrm{bc} / \mathrm{a} \bar{b} / \mathrm{ab}$ & D4S 127 & b/ab/ab \\
\hline D2S2192 & $\mathrm{a} / \mathrm{a} c / \bar{b} \mathrm{c}$ & $\mathrm{D} 2 \mathrm{~S} 2189$ & $\mathrm{bc} / \mathrm{a} \bar{b} / \mathrm{ac}$ & $\mathrm{D} 4 \mathrm{~S} 3023$ & $\mathrm{~b} / \mathrm{ab} c / \mathrm{ac}$ \\
\hline D2S325 & $a b / a b / a b$ & $\mathrm{D} 2 \mathrm{~S} 355$ & $\mathrm{ab} / \mathrm{b} / \mathrm{ab}$ & D4S1599 & $\mathrm{b} / \overline{\mathrm{a}} \mathrm{b} / \mathrm{ac}$ \\
\hline $\mathrm{D} 2 \mathrm{~S} 2274$ & $\mathrm{~b} / \mathrm{ab} / \mathrm{ab}$ & D2S325 & $\mathrm{ab} / \mathrm{bc} / \mathrm{ac}$ & D4S403 & $\mathrm{ab} / \mathrm{ab} / \mathrm{b}$ \\
\hline D2S2242 & $\mathrm{ac} / \mathrm{bc} / \mathrm{ab}$ & $\mathrm{D} 2 \mathrm{~S} 2208$ & $\mathrm{ac} / \mathrm{ab} / \mathrm{bc}$ & D4S1546 & $\mathrm{cd} / a b \mathrm{~d} / \mathrm{ab}$ \\
\hline D2S2178 & $\mathrm{ac} / \mathrm{bc} / \mathrm{b}$ & D2S2274 & $\mathrm{a} / a \overline{\mathrm{b}} / \mathrm{bc}$ & D4S391 & $\mathrm{ac} / \overline{\mathrm{ab}} / \mathrm{bd}$ \\
\hline D2S1385 & $\mathrm{ac} / \mathrm{ac} / \mathrm{bc}$ & $\mathrm{D} 2 \mathrm{~S} 2208$ & $\mathrm{ac} / \mathrm{ab} / \mathrm{bc}$ & $\mathrm{D} 4 \mathrm{~S} 2950$ & $\mathrm{ab} / \mathrm{ab} / \mathrm{b}$ \\
\hline D2S1369 & $\mathrm{ab} / \mathrm{a} / \mathrm{ab}$ & D2S2178 & $\mathrm{ab} / \mathrm{bc} / \mathrm{c}$ & D4S 1627 & $\mathrm{ac} / \mathrm{ab} / \mathrm{bd}$ \\
\hline $\mathrm{D} 2 \mathrm{~S} 157$ & $\mathrm{bd} / \mathrm{bc} / \mathrm{ac}$ & $\mathrm{D} 2 \mathrm{~S} 1385$ & $\mathrm{ab} / \mathrm{bc} / \mathrm{bc}$ & & \\
\hline D2S1380 & $\mathrm{a} / \mathrm{a} / \mathrm{a}$ & D2S1369 & $\mathrm{ab} / \mathrm{ab} / \mathrm{ab}$ & & \\
\hline $\mathrm{D} 2 \mathrm{~S} 1345$ & $\mathrm{ad} / \mathrm{cd} / \mathrm{bc}$ & $\mathrm{D} 2 \mathrm{~S} 157$ & $\mathrm{ab} / \mathrm{ab} / \mathrm{ab}$ & & \\
\hline D2S164 & $\mathrm{ac} / \overline{\mathrm{a}} \mathrm{c} / \mathrm{bc}$ & D2S371 & $\mathrm{bc} / \mathrm{bc} / \mathrm{ac}$ & & \\
\hline D2S163 & $\mathrm{ab} / \mathrm{a} \overline{\mathrm{b}} / \mathrm{ab}$ & $\mathrm{D} 2 \mathrm{~S} 1380$ & $\mathrm{ad} / \mathrm{ac} / \mathrm{bc}$ & & \\
\hline $\mathrm{PAX} 3$ & $\mathrm{a} / \mathrm{a} / \mathrm{a}$ & $\mathrm{D} 2 \mathrm{~S} 143$ & $\mathrm{ac} / \mathrm{bc} / \mathrm{bc}$ & & \\
\hline D2S1363 & $\mathrm{b} / \underline{a b} / \mathrm{ab}$ & $\mathrm{D} 2 \mathrm{~S} 163$ & $\mathrm{ab} / \mathrm{ab} / \mathrm{ab}$ & & \\
\hline D2S159 & $\mathrm{ab} / \mathrm{b} / \mathrm{ab}$ & D2S120 & $\mathrm{ab} / \mathrm{ab} / \mathrm{bc}$ & & \\
\hline $\mathrm{D} 2 \mathrm{~S} 172$ & $\mathrm{ab} / \mathrm{ab} / \mathrm{ab}$ & $\mathrm{D} 2 \mathrm{~S} 126$ & $\mathrm{~cd} / \mathrm{a} d / \mathrm{ab}$ & & \\
\hline $\mathrm{D} 2 \mathrm{~S} 206$ & $\mathrm{ab} / \mathrm{b} c / \mathrm{cd}$ & $\mathrm{D} 2 \mathrm{~S} 159$ & $\mathrm{bc} / \mathrm{ab} / \mathrm{ab}$ & & \\
\hline $\mathrm{D} 2 \mathrm{~S} 345$ & $\mathrm{ab} / \mathrm{a} \bar{b} / \mathrm{b}$ & $\mathrm{D} 2 \mathrm{~S} 172$ & $\mathrm{abb} / \mathrm{ab} / \mathrm{ab}$ & & \\
\hline $\mathrm{D} 2 \mathrm{~S} 125$ & $\mathrm{a} / \mathrm{a} b / \mathrm{b}$ & $\mathrm{D} 2 \mathrm{~S} 206$ & $\mathrm{ab} / \mathrm{ac} / \mathrm{ac}$ & & \\
\hline & & D2S345 & $\mathrm{b} / \mathrm{a} b / \mathrm{a}$ & & \\
\hline
\end{tabular}

46,XX,dup (4) (q25q33)mat

46,XX,dup(5) (q22q35)mat

46,XY,dup (6) (q21q22) mat

\begin{tabular}{|c|c|}
\hline Marker & $M / P / F$ \\
\hline $\begin{array}{l}\text { D4S427 } \\
\text { D4S194 } \\
\text { D4S1615 } \\
\text { D4S175 } \\
\text { D4S2939 } \\
\text { D4S413 } \\
\text { D4S1566 } \\
\text { D4S1552 }\end{array}$ & $\begin{array}{l}\mathrm{b} / \mathrm{ab} / \mathrm{ac} \\
\mathrm{ad} / / \overline{\mathrm{c} d} d / \mathrm{bc} \\
\mathrm{bd} / \mathrm{a} b \bar{d} / \mathrm{ac} \\
\mathrm{ac} / \mathrm{ac} / \mathrm{ab} \\
\mathrm{b} / \mathrm{ab} / \mathrm{ab} \\
\mathrm{ab} / \mathrm{abd} / \mathrm{cd} \\
\mathrm{ab} / \overline{a b} \mathrm{c} / \mathrm{ac} \\
\mathrm{ac} / \mathrm{bc} / \mathrm{ab}\end{array}$ \\
\hline \multicolumn{2}{|c|}{$46, X Y, d u p(5)(p 14 p 15.3) / 46, X Y$} \\
\hline Marker & $M / P / F$ \\
\hline $\begin{array}{l}\text { D5S406 } \\
\text { D5S208 } \\
\text { D5S807 } \\
\text { D5S1963 } \\
\text { D5S1473 } \\
\text { D5S502 } \\
\text { D5S663 }\end{array}$ & $\begin{array}{l}a / a b / b \\
a d / a b / b c \\
a b / b / b \\
a / a b / b \\
b / a b / a \\
a c / a / a \\
a b / b c / c\end{array}$ \\
\hline
\end{tabular}

\begin{tabular}{|c|c|c|c|}
\hline Marker & $M / P / F$ & Marker & $M / P / F$ \\
\hline D5S346 & $\mathrm{bc} / \mathrm{ac} / \mathrm{ab}$ & D6S430 & $\mathrm{bc} / \mathrm{a} c / \mathrm{ab}$ \\
\hline D5S2098 & $\mathrm{bc} / \mathrm{a} \bar{b} c / \mathrm{ac}$ & D6S251 & $\mathrm{ab} / \mathrm{ac} / \mathrm{cd}$ \\
\hline D5S2057 & $a / a b / b$ & D6S275 & $\mathrm{ac} / \mathrm{ac} / \mathrm{bc}$ \\
\hline D5S816 & $\mathrm{ac} / \mathrm{ac} / \mathrm{bc}$ & D6S300 & $\mathrm{ab} / \mathrm{ac} / \mathrm{bc}$ \\
\hline D5S393 & $\mathrm{ab} / \mathrm{ab} / \mathrm{a}$ & D6S252 & $a b / b / b$ \\
\hline D5S673 & $a b / a b / b c$ & D6S434 & $\mathrm{bc} / \mathrm{bc} / \mathrm{ac}$ \\
\hline D5S820 & $\mathrm{bc} / \mathrm{a} b c / \mathrm{ac}$ & D6S268 & $\mathrm{cd} / \overline{\mathrm{b}} d / \mathrm{ab}$ \\
\hline D5S423 & $\mathrm{bd} / \mathrm{a} \overline{b d} / \mathrm{ac}$ & D6S302 & $\mathrm{c} / \mathrm{a} c / \bar{a} \mathrm{~b}$ \\
\hline D5S400 & $\mathrm{a} / \mathrm{a} / \mathrm{ab}$ & D6S261 & $\mathrm{bc} / \overline{\mathrm{b}} c / \mathrm{ab}$ \\
\hline D5S1456 & $a b / a b / b c$ & D6S407 & $\mathrm{ab} / \underline{b} \bar{c} / \mathrm{ac}$ \\
\hline D5S429 & $\mathrm{ac} / \mathrm{ab} c / \mathrm{ab}$ & D6S262 & $\mathrm{ab} / \overline{\mathrm{ac}} / \mathrm{c}$ \\
\hline D5S498 & $\mathrm{ab} / \overline{\mathrm{a}} \mathrm{b} / \mathrm{b}$ & D6S270 & $\mathrm{ac} / \mathrm{ac} / \mathrm{bc}$ \\
\hline D5S2006 & $a b / b / b$ & D6S292 & $\mathrm{ac} / \mathrm{bc} / \mathrm{bd}$ \\
\hline
\end{tabular}

D5S2006 ab/b/b

D6S292

$\mathrm{ac} / \mathrm{bc} / \mathrm{bd}$

\begin{tabular}{|c|c|c|c|c|c|c|}
\hline \multicolumn{2}{|c|}{$46, X Y, d u p(7)(q 22 q 31) m a t$} & \multicolumn{3}{|c|}{$\begin{array}{l}\text { 46,XX,dup (8) (p10p23)mat, } \\
\operatorname{del}(8)(p 23 p 23.3) m a t\end{array}$} & \multicolumn{2}{|c|}{$46, X X, d u p(8)(q 22 q 24.3) p a t$} \\
\hline Marker & $M / P / F$ & Marker & $M / P / F$ & Deletion & Marker & $M / P / F$ \\
\hline D7S527 & $\mathrm{cd} / \mathrm{a} c / \mathrm{ab}$ & D8S264 & $\mathrm{a} / \mathrm{c} / \mathrm{bc}$ & mat del & $\mathrm{D} 8 \mathrm{~S} 273$ & $\mathrm{ab} / \mathrm{ab} / \mathrm{ac}$ \\
\hline D7S515 & $\mathrm{ab} / \mathrm{b} / \mathrm{bc}$ & D8S262 & $\mathrm{bc} / \mathrm{a} / \mathrm{ab}$ & mat del & D8S559 & $\mathrm{ab} / \mathrm{a} b / \mathrm{b}$ \\
\hline D7S496 & $\mathrm{bc} / \mathrm{b} c / \mathrm{ab}$ & D8S1824 & $\mathrm{ac} / \mathrm{a} / \mathrm{ab}$ & ? & D8S521 & $a b / a \bar{b} / a$ \\
\hline D7S501 & $\mathrm{ab} / \mathrm{ab} / \mathrm{b}$ & D8S1806 & $a / b / b$ & mat del & D8S556 & $\mathrm{b} / \mathrm{ab} / \mathrm{a}$ \\
\hline D7S523 & $a b / a b / a b$ & D8S201 & $\mathrm{b} / \mathrm{b} / \mathrm{ab}$ & ? & D8S281 & $\mathrm{ac} / \mathrm{a} b / \mathrm{b}$ \\
\hline D7S486 & $\mathrm{b} / b \mathrm{c} / \mathrm{ac}$ & D8S307 & $a b / b / b c$ & $?$ & D8S522 & $\mathrm{bc} / \mathrm{a} \bar{b} / \mathrm{ab}$ \\
\hline D7S522 & $\mathrm{a} / \overline{\mathrm{a}} / \mathrm{ab}$ & D8S439 & $\mathrm{ab} / \mathrm{b} / \mathrm{bc}$ & ? & D8S514 & $\mathrm{a} / \mathrm{a} b / \mathrm{b}$ \\
\hline CF-108 & $\mathrm{b} / \mathrm{b} / \mathrm{ab}$ & D8S552 & $\mathrm{a} / \mathrm{ab} / \mathrm{b}$ & & D8S266 & $\mathrm{bc} / \bar{a} \mathrm{~b} / \mathrm{ab}$ \\
\hline CF-332 & $\mathrm{c} / \mathrm{b} c / \mathrm{ab}$ & D8S133 & $\mathrm{bc} / \mathrm{bc} / \mathrm{ac}$ & & D8S508 & $\mathrm{b} / \mathrm{b} / \mathrm{b}$ \\
\hline CF-333 & $a b / a b / b$ & D8S261 & $a b / a b / b$ & & D8S263 & $\mathrm{a} / \mathrm{ac} / \mathrm{bc}$ \\
\hline D7S495 & $\mathrm{bc} / \mathrm{b} / \mathrm{ac}$ & D8S1145 & $\mathrm{a} / \mathrm{ab} / \mathrm{b}$ & & D8S557 & $\mathrm{a} / \mathrm{a} / \mathrm{a}$ \\
\hline D7S483 & $\mathrm{bc} / \mathrm{a} b / \mathrm{ab}$ & LPL & $\mathrm{ab} / \mathrm{abc} / \mathrm{bc}$ & & D8S529 & $\mathrm{ac} / \mathrm{bc} / \mathrm{b}$ \\
\hline & & NEFL & $\mathrm{bc} / \overline{\mathrm{bc}} / \mathrm{ac}$ & & D8S256 & $\mathrm{b} / \mathrm{ab} / \mathrm{ab}$ \\
\hline & & D8S505 & $\mathrm{ab} / \mathrm{ab} / \mathrm{bc}$ & & D8S1746 & $\mathrm{bc} / \mathrm{ac} / \mathrm{ac}$ \\
\hline & & D8S322 & $\mathrm{bc} / \underline{b} \mathrm{c} / \mathrm{ac}$ & & D8S1710 & $\mathrm{ab} / \mathrm{a}-\mathrm{c}$ \\
\hline & & D8S136 & $\mathrm{ab} / \mathrm{ab} / \mathrm{bc}$ & & D8S537 & $\mathrm{ac} / \mathrm{a} \bar{c} / \mathrm{bc}$ \\
\hline & & D8S137 & $\mathrm{b} / \mathrm{b} / \mathrm{ab}$ & & D8S272 & $\mathrm{ab} / \overline{\mathrm{b}} \mathrm{c} / \mathrm{bc}$ \\
\hline & & D8S87 & $\mathrm{ab} / \mathrm{ab} / \mathrm{ab}$ & & D8S274 & $\mathrm{ab} / \mathrm{a} \overline{\mathrm{b}} / \mathrm{ab}$ \\
\hline & & ANK1 & $\mathrm{a} / \mathrm{a} / \mathrm{a}$ & & & \\
\hline & & PLAT & $\mathrm{ac} / \mathrm{ac} / \mathrm{ab}$ & & & \\
\hline
\end{tabular}

Results of analysis of microsatellite markers of the duplicated regions from all investigated cases. Markers fully or in part informative are underlined and in italics. Markers are ordered from $\mathrm{p}$ to $\mathrm{q}$ telomere. $\mathrm{M}=$ mother, $\mathrm{P}=$ patient, $\mathrm{F}=$ father. 
Table 2 Results of investigated markers mapping to the duplicated regions (continued)

\begin{tabular}{|c|c|c|c|c|c|c|}
\hline \multicolumn{2}{|c|}{$46, X Y, d u p(9)(p 13 p 24) p a t$} & \multicolumn{3}{|c|}{$46, X X, d u p(9)(p 13 p 24) p a t$} & \multicolumn{2}{|c|}{$46, X X, d u p(10)(q 22 q 26)$} \\
\hline Marker & $M / P / F$ & Marker & \multicolumn{2}{|l|}{$M / P / F$} & \multirow{2}{*}{$\begin{array}{l}\text { Marker } \\
\text { D10S195 }\end{array}$} & $M / P / F$ \\
\hline D9S1779 & $\mathrm{ab} / \mathrm{a} c / \mathrm{c}$ & D9S178 & ab/ab/a & & & $\mathrm{a} / \mathrm{a} / \mathrm{ab}$ \\
\hline D9S132 & b/ab/ab & D9S132 & $\mathrm{b} / \mathrm{b} / \mathrm{ab}$ & & D10S2327 & $\mathrm{b} / \mathrm{b} / \mathrm{ab}$ \\
\hline D9S144 & $\mathrm{b} / \mathrm{b} / \mathrm{ab}$ & D9S144 & c/ac/ab & & D10S1686 & $\mathrm{ac} / \mathrm{ac} / \mathrm{bc}$ \\
\hline D9S256 & $\mathrm{ac} / a \mathrm{c} / \mathrm{ab}$ & D9S256 & $\mathrm{a} / \mathrm{a} / \mathrm{a}$ & & D10S215 & $\mathrm{a} / \mathrm{a} / \mathrm{a}$ \\
\hline D9S269 & $\mathrm{a} / \mathrm{a} / \mathrm{ab}$ & D9S269 & $\mathrm{ac} / \underline{b c} / \mathrm{ab}$ & & D10S677 & $\mathrm{ab} / \mathrm{bc} / \mathrm{cd}$ \\
\hline D9S156 & $\mathrm{b} / \mathrm{b} \underline{c} / \mathrm{ac}$ & D9S285 & $\mathrm{bc} / \underline{b} \mathrm{c} / \mathrm{ab}$ & & D10S534 & $\mathrm{ab} / \mathrm{ab} / \mathrm{ab}$ \\
\hline D9S157 & $\mathrm{ac} / \overline{\mathrm{c}} d / \mathrm{bd}$ & D9S156 & $\mathrm{ab} / \mathrm{a} b / \mathrm{b}$ & & D10S562 & $\mathrm{ab} / \mathrm{a} / \mathrm{ab}$ \\
\hline D9S171 & $\mathrm{ab} / \mathrm{a} \bar{b} / \mathrm{bc}$ & D9S157 & $\mathrm{ab} / \mathrm{a} \bar{b} / \mathrm{bc}$ & & D10S1237 & $\mathrm{a} / \mathrm{a} / \mathrm{a}$ \\
\hline D9S161 & $\mathrm{b} / \underline{a} \bar{b}-\mathrm{ac}$ & D9S162 & $a b / a \bar{b} / b c$ & & D10S209 & $a b / a / a$ \\
\hline D9S1868 & $\mathrm{a} / \bar{a} \bar{b} / \mathrm{a} b$ & D9S171 & $\mathrm{b} / \mathrm{b} / \mathrm{ab}$ & & D10S1230 & $\mathrm{ac} / \mathrm{bc} / \mathrm{bc}$ \\
\hline \multirow{5}{*}{ D9S304 } & $\mathrm{ab} / \mathrm{a} / \mathrm{ab}$ & D9S161 & $\mathrm{ab} / \mathrm{a} \underline{c} / \mathrm{cd}$ & & D10S1213 & $\mathrm{ab} / \mathrm{bc} / \mathrm{bc}$ \\
\hline & & D9S1868 & $\mathrm{ac} / \mathrm{bc} / \mathrm{b}$ & & D10S590 & $\mathrm{a} / \mathrm{a} / \mathrm{a}$ \\
\hline & & D9S104 & $\mathrm{ac} / \mathrm{a} b / \mathrm{b}$ & & D10S212 & $\mathrm{a} / \mathrm{a} / \mathrm{ab}$ \\
\hline & & D9S304 & $\mathrm{bc} / \mathrm{b} \bar{d} / \mathrm{ad}$ & & & \\
\hline & & D9S200 & $\mathrm{b} / \underline{a b} \overline{\mathrm{a}} \mathrm{ab}$ & & & \\
\hline \multicolumn{2}{|c|}{$46, X X, d u p(11)(q 13.2 q 13.4) p a t$} & \multicolumn{3}{|c|}{$46, X X, d u p(12)(q 22 q 24.3)$} & \multicolumn{2}{|c|}{$46, X X, \operatorname{dup}(14)(q 21 q 21)$} \\
\hline Marker & $M / P / F$ & Marker & $M / P / F$ & & Marker & $M / P / F$ \\
\hline D11S4191 & $\mathrm{ac} / \mathrm{a} b c / \mathrm{bc}$ & D12S1722 & $a b / b / b$ & & $\mathrm{D} 14 \mathrm{~S} 80$ & $\mathrm{bc} / \mathrm{bc} / \mathrm{ab}$ \\
\hline D11S534 & $\mathrm{ab} / \mathrm{ac} / \mathrm{ac}$ & D12S43 & $\mathrm{b} / \mathrm{b} / \mathrm{ba}$ & & D14S70 & $\mathrm{bc} / \mathrm{bc} / \mathrm{ab}$ \\
\hline D11S916 & $\mathrm{b} / \mathrm{bc} / \mathrm{ac}$ & D12S81 & $a b / a b / a b$ & & D14S75 & $\mathrm{ac} / \mathrm{ac} / \mathrm{bc}$ \\
\hline D11S527 & $\mathrm{bc} / \mathrm{ac} / \mathrm{ab}$ & $\mathrm{D} 12 \mathrm{~S} 1660$ & $\mathrm{c} / \mathrm{ac} / \mathrm{ab}$ & & D14S288 & b/ab/ac \\
\hline D11S2002 & $\mathrm{ab} / \underline{a} \mathrm{~b} \underline{c} / \mathrm{ac}$ & D12S1588 & $\mathrm{ab} / \mathrm{ac} / \mathrm{bc}$ & & D14S989 & $\mathrm{b} / \mathrm{ab} / \mathrm{ab}$ \\
\hline D11S901 & $\mathrm{ac} / \overline{\mathrm{ac}} / \overline{\mathrm{a}} \mathrm{b}$ & IGFI & $\mathrm{ab} / \mathrm{b} / \mathrm{b}$ & & D14S1057 & $a b / a b / a b$ \\
\hline \multirow[t]{12}{*}{ D11S876 } & $\mathrm{bc} / \mathrm{bc} / \mathrm{ac}$ & D12S84 & $\mathrm{b} / \mathrm{ab} / \mathrm{a}$ & & D14S276 & $\mathrm{ab} / \mathrm{ab} / \mathrm{ab}$ \\
\hline & & D12S79 & $\mathrm{a} / \mathrm{a} / \mathrm{a}$ & & D14S1064 & $\mathrm{b} / \mathrm{ab} / \mathrm{ab}$ \\
\hline & & $\mathrm{D} 12 \mathrm{~S} 342$ & $a b / a b / a b$ & & D14S274 & $\mathrm{ac} / \mathrm{bc} / \mathrm{bc}$ \\
\hline & & D12S1675 & $\mathrm{ab} / \mathrm{b} / \mathrm{b}$ & & D14S750 & $\mathrm{b} / \mathrm{ab} / \mathrm{ac}$ \\
\hline & & D12S1679 & $\mathrm{ac} / \mathrm{ab} / \mathrm{b}$ & & D14S290 & $\mathrm{ab} / \mathrm{ab} / \mathrm{bc}$ \\
\hline & & D12S834 & $\mathrm{a} / \mathrm{ab} / \mathrm{ab}$ & & D14S63 & $a b / a b / b c$ \\
\hline & & D12S1714 & $\mathrm{b} / \mathrm{ab} / \mathrm{ab}$ & & D14S77 & $\mathrm{bc} / \mathrm{ab} / \mathrm{a}$ \\
\hline & & $\mathrm{D} 12 \mathrm{~S} 1723$ & $\mathrm{~b} / \mathrm{b} / \mathrm{ab}$ & & D14S42 & $\mathrm{cd} / \mathrm{ac} / \mathrm{ab}$ \\
\hline & & D12S343 & $\mathrm{a} / \mathrm{a} / \mathrm{ab}$ & & D14S67 & $\mathrm{ac} / \mathrm{ac} / \mathrm{bc}$ \\
\hline & & D12S1638 & $a / a b / b$ & & D14S68 & $\mathrm{ab} / \mathrm{bc} / \mathrm{bc}$ \\
\hline & & D12S357 & $\mathrm{a} / \mathrm{a} / \mathrm{a}$ & & D14S51 & $\mathrm{ac} / \mathrm{ab} / \mathrm{ab}$ \\
\hline & & & & & D14S62 & $\mathrm{ab} / \mathrm{ac} / \mathrm{c}$ \\
\hline \multicolumn{2}{|c|}{$46, X Y, d u p(15)(q 13 q 13)$} & $\begin{array}{l}46, X X, d u p \\
\text { (q13q13), de }\end{array}$ & $\begin{array}{l}(q 11 q 13) m \\
(q 21 q 21)\end{array}$ & (19) & $46, X Y, d u p$ & (q11.1q13.1) \\
\hline Marker & $M / P / F$ & Marker & $M / P / F$ & Deletion & Marker & $M / P / F$ \\
\hline D15S113 & $\mathrm{a} / \mathrm{a} / \mathrm{a}$ & -LIPE & $\mathrm{ab} / \mathrm{ab} / \mathrm{bc}$ & & $\mathrm{D} 22 \mathrm{~S} 264$ & $\mathrm{ab} / \mathrm{bc} / \mathrm{bc}$ \\
\hline D15S122 & $\mathrm{ab} / \mathrm{ac} / \mathrm{c}$ & D19S191 & $\mathrm{bc} / \mathrm{bc} / \mathrm{ab}$ & & $\mathrm{D} 22 \mathrm{~S} 343$ & $\mathrm{a} / \mathrm{ac} / \mathrm{bc}$ \\
\hline GABR3 & $\mathrm{ab} / \mathrm{bc} / \mathrm{c}$ & D19S223 & $\mathrm{a} / \mathrm{ab} / \mathrm{b}$ & & D22S427 & $\mathrm{b} / \mathrm{bc} / \mathrm{ac}$ \\
\hline D15S217 & $\mathrm{ac} / \mathrm{ab} / \mathrm{bc}$ & D19S900 & $a b / a b / b c$ & & $\mathrm{D} 22 \mathrm{~S} 941$ & $\mathrm{bc} / \mathrm{b} / \mathrm{ab}$ \\
\hline D15S541 & $\mathrm{ab} / \mathrm{b} / \mathrm{ab}$ & D19S913 & $\mathrm{ac} / \mathrm{ab} / \mathrm{ab}$ & & D22S944 & $\mathrm{ab} / \mathrm{ab} / \mathrm{ab}$ \\
\hline D15S542 & $\mathrm{bd} / \mathrm{cd} / \mathrm{ac}$ & D19S219 & $\mathrm{a} / \mathrm{ab} / \mathrm{bc}$ & & $\mathrm{D} 22 \mathrm{~S} 351$ & $\mathrm{ab} / \mathrm{bc} / \mathrm{bc}$ \\
\hline D15S97 & $\mathrm{ab} / \mathrm{ab} / \mathrm{ac}$ & MD & $\mathrm{ac} / \mathrm{ac} / \mathrm{bc}$ & & D22S1638 & $\mathrm{bc} / \mathrm{ac} / \mathrm{ac}$ \\
\hline D15S11 & $\mathrm{ab} / \mathrm{ac} / \mathrm{c}$ & APOC2 & $\mathrm{bc} / \mathrm{ab} / \mathrm{ab}$ & & D22S311 & $\mathrm{ad} / \mathrm{ab} / \mathrm{bc}$ \\
\hline & & D19S412 & $\mathrm{bc} / \mathrm{bc} / \mathrm{ab}$ & & $\mathrm{D} 22 \mathrm{~S} 306$ & $a b / a b / b c$ \\
\hline & & D19S112 & $\mathrm{ab} / \mathrm{ab} / \mathrm{bc}$ & & $\mathrm{D} 22 \mathrm{~S} 303$ & $\mathrm{~b} / \mathrm{ab} / \mathrm{a}$ \\
\hline $46, X X, d u p($ & p13)pat & D19S866 & $\mathrm{ab} / \mathrm{a} / \mathrm{ac}$ & $?$ & $\mathrm{D} 22 \mathrm{~S} 258$ & $\mathrm{a} / \mathrm{a} / \mathrm{a}$ \\
\hline Marker & $M / P / F$ & D19S574 & $\mathrm{ac} / \mathrm{b} / \mathrm{b}$ & mat del & $\mathrm{D} 22 \mathrm{~S} 315$ & $\mathrm{ab} / \mathrm{ab} / \mathrm{bc}$ \\
\hline D17S1866 & $\mathrm{ac} / \mathrm{a} / \mathrm{ab}$ & D19S246 & $\mathrm{bc} / \mathrm{a} / \mathrm{ac}$ & mat del & $\mathrm{D} 22 \mathrm{~S} 277$ & $\mathrm{a} / \mathrm{ac} / \mathrm{bc}$ \\
\hline D17S926 & $\mathrm{ab} / \mathrm{bc} / \mathrm{cd}$ & D19S206 & $\mathrm{b} / \mathrm{a} / \mathrm{ac}$ & mat del & $\mathrm{D} 22 \mathrm{~S} 274$ & $\mathrm{ac} / \mathrm{bc} / \mathrm{b}$ \\
\hline D17S938 & $\mathrm{ac} / \mathrm{a} / \mathrm{ab}$ & D19S888 & $\mathrm{b} / \mathrm{c} / \mathrm{ac}$ & mat del & & \\
\hline D17S520 & $\mathrm{ab} / \mathrm{a} b / \mathrm{bc}$ & D19S180 & $\mathrm{ac} / \mathrm{b} / \mathrm{ab}$ & mat del & & \\
\hline D17S1875 & $\mathrm{ab} / \overline{\mathrm{b}} \underline{\mathrm{c}} / \mathrm{cd}$ & D19S572 & $\mathrm{ac} / \mathrm{b} / \mathrm{b}$ & mat del & & \\
\hline D17S969 & $a b / a / a$ & D19S254 & $\mathrm{ac} / \mathrm{b} / \mathrm{ab}$ & mat del & & \\
\hline D17S921 & $a b / a b / a b$ & D19S880 & $\mathrm{ab} / \mathrm{b} / \mathrm{ab}$ & $?$ & & \\
\hline $\mathrm{D} 17 \mathrm{~S} 261$ & $\mathrm{a} / \mathrm{ab} / \mathrm{ab}$ & D19S877 & $\mathrm{a} / \mathrm{a} / \mathrm{a}$ & $?$ & & \\
\hline CHRNB1 & $\mathrm{bc} / \mathrm{c} / \mathrm{ac}$ & D19S720 & $\mathrm{a} / \mathrm{a} / \mathrm{a}$ & $?$ & & \\
\hline D17S122 & $\mathrm{b} / \underline{a} \mathrm{~b} \underline{c} / \mathrm{ac}$ & D19S210 & $\mathrm{ab} / \mathrm{a} / \mathrm{ab}$ & $?$ & & \\
\hline
\end{tabular}

\section{Discussion}

We report on cytogenetic, FISH, and molecular findings in 20 cases with de novo direct or inverted tandem duplications of 18 different autosomal chromosome segments. "Tandem duplication" is a cytogenetic term and refers to the direct or inverted order of cytogenetically recognisable genetic material one after the other. ${ }^{2}$ Mechanisms of formation are not known. In theory, the duplication might be direct or inverted, either within the same chromatid (A, B) or between non-sister chromatids (C, D) during replication or by unequal crossover (fig 3). ${ }^{2}$ The rearrangement between non-sister chromatids can also be regarded as an insertion within the same chromosome arm. Definitive discrimination is not possible by standard techniques. Molecular methods like Southern blotting or microsatellite analysis as well as FISH are only able to show the presence or absence of an allele or genetic material, but not the exact localisation of a specific allele. It is not possible to differentiate physical localisation of maternal and paternal alleles by these methods. Therefore, the results and relevance of our study and of previously reported cases are limited. For an exact evaluation it would be necessary to separate the aberrant chromosome 

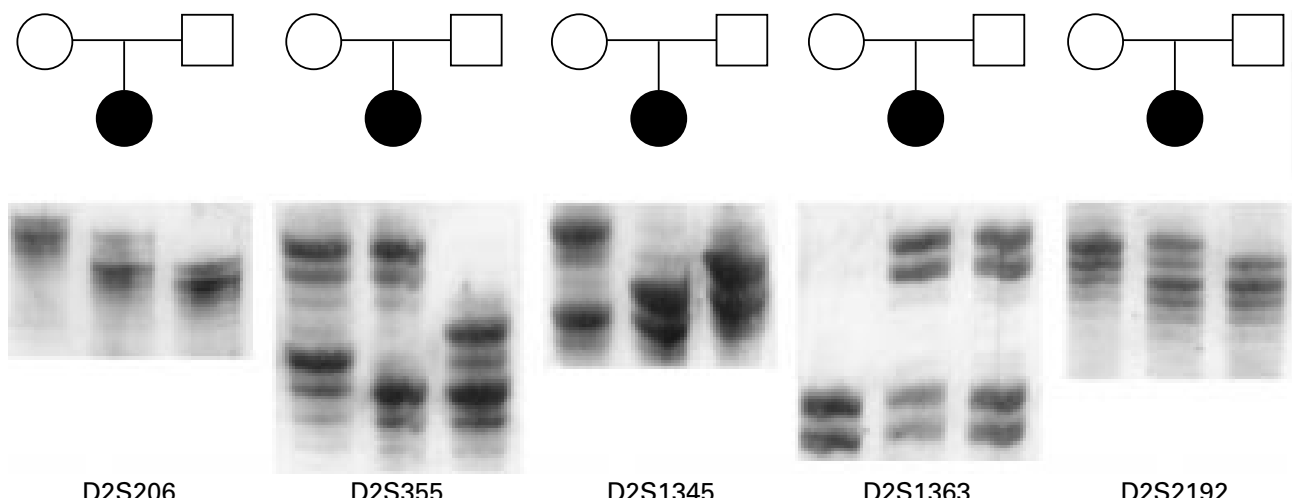

D2S1345
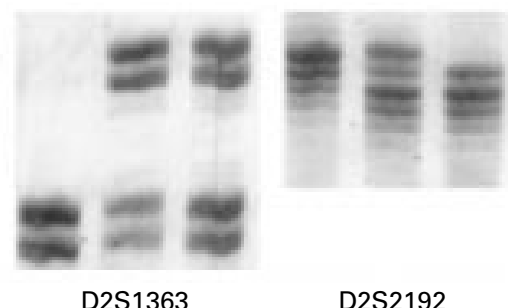

D2S2192

Figure 1 Molecular findings in case 1 (46,XX,dup(2)(q35q37)pat)) showing sister chromatid rearrangement by one maternal allele and one more intense paternal allele at each of the microsatellite markers investigated, D2S355, D2S2192, D2S1345, D2S1363, and D2S206.

from its homologue and afterwards to investigate markers located within the duplication. Only by this approach would it be possible to indicate the exact physical localisation of each allele either on the paternal or on the maternal homologue and the mechanism of formation dependent on the constellation of the two alleles.

Another problem of our study and of other published cases is the lack of data on the exact molecular breakpoints. Breakpoints determined either by conventional cytogenetic methods or by FISH with specific cosmids may differ by several megabases. What seems identical cytogenetically might be completely different at the molecular level. Therefore, the smaller the duplicated region, the less likely an informative result will be obtained.

Comparing the minimum 43 breakpoints in our cases with 150 common or rare fragile sites reported recently, ${ }^{14} 23$ breakpoints (53\%) were localised to the same cytogenetic band as common or rare fragile sites (underlined in table 1). This is clearly more than the number of 13 breakpoints localised to the same cytogenetic band as common or rare fragile sites expected on a level of 500 bands. In two cases with direct duplications and in all three cases with obviously inverted duplications, both break-
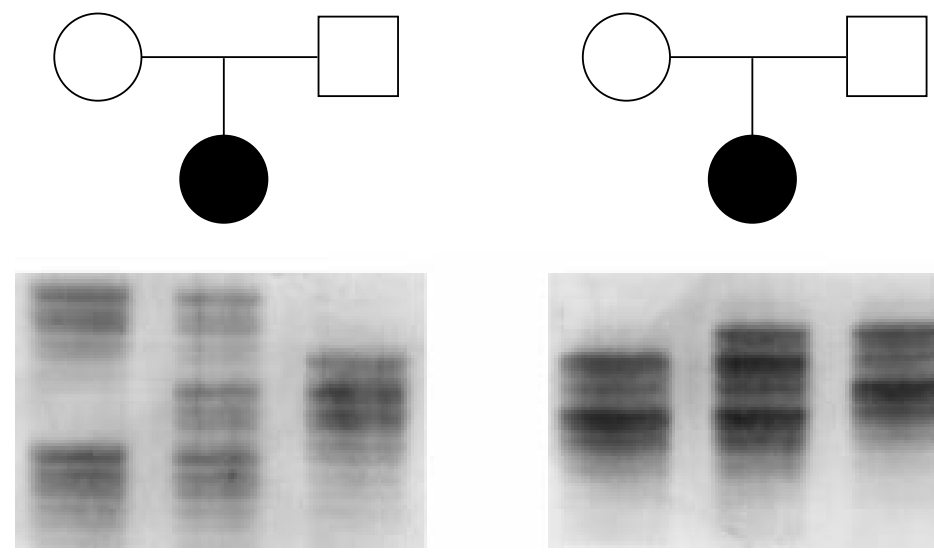

D4S194

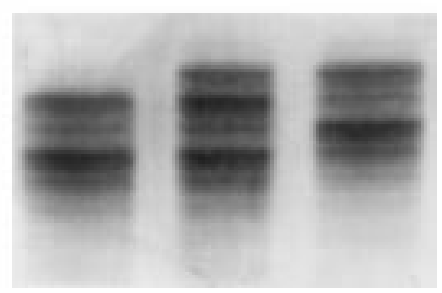

D4S1615

Figure 2 Molecular findings in case 4 (46,XX,dup(4)(q25q33)mat) showing non-sister chromatid rearrangement during maternal meiosis by two maternal alleles and one paternal allele at each of the investigated microsatellite markers D4S194 and D4S1615. points mapped to common fragile sites. ${ }^{14}$ As a consequence, an unequal crossover may be more likely. From these observations, it could be suggested that fragile sites are susceptible not only to breakage and subsequent deletions, but can finally result in direct or inverted duplications too.

Another way to explain deletions or duplications is a simple misalignment of homologous sequences. As recently shown in CharcotMarie-Tooth type 1A neuropathy, duplications of approximately $1.5 \mathrm{Mb}$ are the reciprocal products of homologous recombination events between misaligned flanking CMT1A-REP repeats..$^{15}$ The same mechanism is assumed in microdeletions such as Williams-Beuren syndrome or CATCH $22 .^{16}$

Both SCR and N-SCR may also be found in mosaic cases. Three alleles always suggest meiotic formation and N-SCR. However, three alleles might also be present in cases formed premeiotically and going through meiosis without SCR or N-SCR. Two alleles are expected in cases formed either during meiosis by SCR or during mitosis by both N-SCR and SCR. In the latter situation a rearrangement between non-sister chromatids cannot be distinguished from a rearrangement between sister chromatids by microsatellite analysis, since there are always only two alleles. Mosaicism can only be detected by different cell lines on chromosome analysis. However, low level mosaicism or mosaicism restricted to the placenta or other uninvestigated tissues may be overlooked. Therefore, there might be more than the one mosaic case (case 5) in our sample. Since, in general, there are few reported cases with mosaicism of direct or inverted duplications, ${ }^{1}$ we assumed meiotic origin in all of our cases without mosaicism in lymphocytes.

In our cases a 2:1 ratio of SCR versus N-SCR and an almost equal distribution of paternal and maternal origin, both in cases with N-SCR and in the informative cases with SCR, was found.

The mode of formation seems to be more complex in some cases. Almost all cases with an inverted duplication (8) (p10p23) are formed 


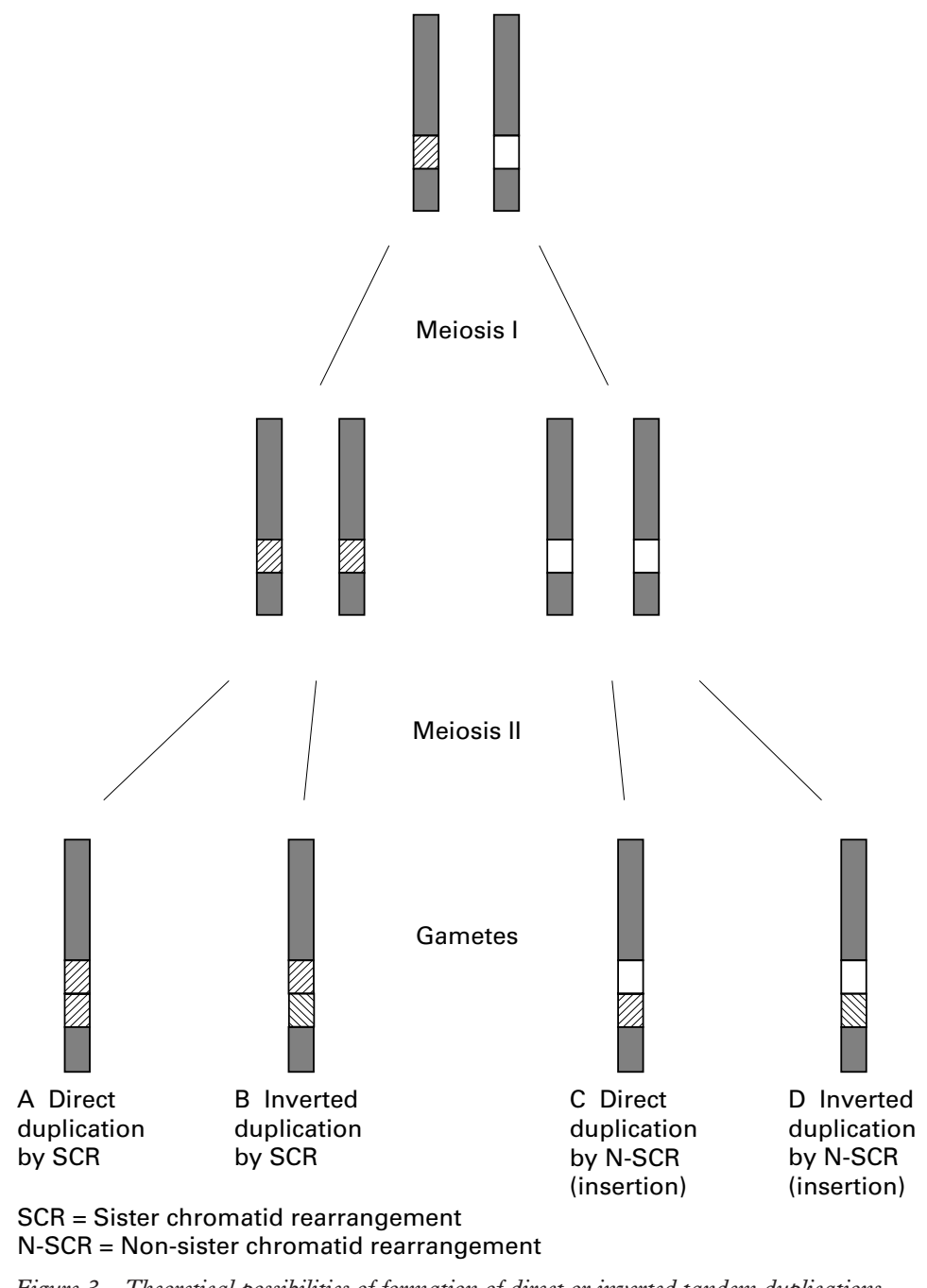

Figure 3 Theoretical possibilities of formation of direct or inverted tandem duplications.

during maternal meiosis and are often associated with a telomeric deletion (8) (p23p23.3). ${ }^{3}$ These observations were confirmed in case 9 of our study. A similar result of a duplication associated with a deletion was obtained in a case with inv $\operatorname{dup}(7)(\mathrm{q} 21.2 \mathrm{q} 36) .^{7}$ Abnormal pairing during maternal meiosis followed by an unequal crossover owing to misalignment of homologous sequences has been assumed. Moreover, in our study we found a concomitant deletion (del(19)(q13q13)) in one case with an interstitial duplication $(\operatorname{dup}(19)$ (q11q13)). The exact mechanism in this case remains unknown. However, based on this observation the phenotype of pure duplications should be interpreted with caution as other cases may also be associated with undetected small deletions.

In conclusion, our study shows that N-SCR and SCR occur in maternal and paternal meiosis with equal frequency, that N-SCR is less frequent than SCR, and that there is a weak association with common or rare fragile sites.

The authors would like to thank all parents and clinicians for data and information as well as for their excellent cooperation. (grant Nos 32-37798.93 and 32-45604.95) and by the Sandoz Foundation (grant No 98B19).

1 Schinzel A. Human cytogenetics database. Oxford Medical Databases Series. Oxford: Oxford University Press, 1994.

2 Van Dyke D. Isochromosomes and interstitial tandem direct and inverted duplications. In: Daniel A, ed. The cytogenetics of mammalian autosomal rearrangements. Vol 21. New York: Alan R Liss, 1988:635-65.

3 Floridia G, Piantanida M, Minelli A, et al. The same molecular mechanism at the maternal meiosis I produces mono- and dicentric 8p duplications. Am 7 Hum Genet 1996;58:785-96.

4 Breslau-Siderius EJ, Wijnen J, Dauwerse JG, de Pater JM, Beemer FA, Khan PM. Paternal duplication of chromosome 5q11.2-5q14 in a male born with craniostenosis, ear tags, kidney dysplasia and several other anomalies. Hum Genet 1993;92:481-5.

5 Eidelman IA, Zlotogora J, Lerer I, Dagan J, Marks K, Abeliovich D. Transient neonatal diabetes in a child with invdup(6)(q22q23) of paternal origin. Eur f Hum Genet 1997;5:417-19.

6 Smith A, Jauch A, Slater H, Robson L, Sandanam T. Syndromal obesity due to paternal duplication 6(q24.3q27). Am F Med Genet 1999;84:125-31.

7 Stetten G, Charity LL, Kasch LM, et al. A paternally derived inverted duplication of $7 \mathrm{q}$ with evidence of a telomeric deletion. Am f Med Genet 1997;68:76-81.

8 Cotter PD, McCurdy LD, Gershin IF, Babu A, Willner JP, Desnick RJ. Prenatal detection and molecular characterization of a de novo duplication of the distal long arm of chromosome 19. Am f Med Genet 1997;71:325-8.

9 Blouin JL, Aurias A, Creau-Goldberg N, et al. Cytogenetic and molecular analysis of a de novo tandem duplication of and molecular analysis of a de novo tandem dup

10 Trautmann U, Pfeiffer RA, Seufert-Satomi U, Tietzte HU. Simultaneous de novo interstitial deletion of $16 \mathrm{q} 21$ and intercalary duplication of $19 \mathrm{q}$ in a retarded infant with minor dysmorphic features. F Med Genet 1993;30:330-1.

11 Kotzot D, Binkert F, Fokstuen S, et al. Origin and formation of cytogenetically recognizable de novo tandem duplications. Medgen 1999;11:4-26.

12 Ning Y, Roschke A, Smith ACM, et al. A complete set of human telomeric probes and their clinical application. Nat Genet 1996;14:86-9.

13 Dutly F, Baumer A, Kayserili H, Yüksel-Apak M, Zerova T, Schinzel A. Seven cases of Wiedemann-Beckwith syndrome, including the first reported case of mosaic paternal isodisomy along the whole chromosome 11. Am $f \mathrm{Med}$ Genet 1998;79:347-53.

14 Simonic I, Gericke GS. The enigma of common fragile sites. Hum Genet 1996;97:524-31.

15 Reiter LT, Hastings PJ, Nelis E, De Jonghe P, Van Broeckhoeven C, Lupski JR. Human meiotic recombination products revealed by sequencing a hotspot for homologous strand exchange in multiple HNPP deletion patients. Am f Hum Genet 1998;62:1023-33.

16 Baumer A, Dutly F, Balmer D, et al. High level of unequal meiotic crossover at the origin of the $22 \mathrm{q} 11.2$ and $7 \mathrm{q} 11.23$ deletions. Hum Mol Genet 1998;7:887-94. 